\title{
Transport and stability studies on high band gap a-Si:H films prepared by argon dilution
}

\author{
PURABI GOGOI ${ }^{1}, \mathrm{P}_{\mathrm{N}}$ DIXIT $^{2}$ and PRATIMA AGARWAL ${ }^{1, *}$ \\ ${ }^{1}$ Department of Physics, Indian Institute of Technology Guwahati, Guwahati 781039 , \\ India \\ ${ }^{2}$ Plasma Processed Materials Division, National Physical Laboratory, New Delhi 110 012, \\ India \\ ${ }^{*}$ Corresponding author. E-mail: pratima@iitg.ernet.in
}

\begin{abstract}
Device quality hydrogenated amorphous silicon films (a-Si:H) are deposited at a high deposition rate (4-5 $\AA / \mathrm{s})$ using a mixture of argon and hydrogen-diluted silane. The films exhibit good opto-electronic properties and show less degradation upon light soaking. Light-induced changes in conductivity could be annealed at much lower temperature. The presence of $\mathrm{Ar}^{*}$ and atomic hydrogen in plasma replaces the weak $\mathrm{Si}-\mathrm{Si}$ bonds, which are responsible for light-induced degradation by strong $\mathrm{Si}-\mathrm{Si}$ bonds. This results in the improved stability of the films.
\end{abstract}

Keywords. Amorphous silicon; argon dilution; stability; deposition rate; Staebler Wronski effect.

PACS Nos 81.05.Gc; 81.15.Gh; 73.61.Jc; 78.30.-J

\section{Introduction}

Thin films of hydrogenated amorphous silicon (a-Si:H) are now being used in large area photovoltaic and other optoelectronic devices [1]. The efficiency of these solar cells however decreases with exposure to light [2]. In order to increase the stability of these films, changes in deposition parameters including the dilution of the precursor gas silane $\left(\mathrm{SiH}_{4}\right)$ with hydrogen or argon have been tried. Whereas, considerably low deposition rate of films prepared using high hydrogen dilution of $\mathrm{SiH}_{4}$ [3] puts a question towards cost-effectiveness of these films, columnar growth of argon dilution films $[4,5]$ reduces the usefulness. Very high hydrogen and argon dilution further results in the polycrystalline/microcrystalline films [6-8].

In this paper, we report structural, transport and stability studies of amorphous silicon films prepared using a mixture of silane, hydrogen and argon as precursor gas. The films reported have very high deposition rate $(4-5 \AA / \mathrm{s})$ compared to that reported in literature. The structural studies suggest these to be amorphous in nature with improved medium range order (MRO) along with presence of very small nanocrystallites. These films show good optoelectronic properties and less 


\section{Purabi Gogoi, P N Dixit and Pratima Agarwal}

Table 1. Deposition condition, thickness and band gap $\left(E_{\mathrm{G}}\right)$ of a-Si:H films used in the present study. The argon dilution ratio was varied by varying silane flow rate in the chamber. Argon dilution ratio $R$ is defined as the ratio of argon flow rate to equivalent pure silane flow rate. All the films were deposited for $20 \mathrm{~min}$.

\begin{tabular}{lccccc}
\hline Sample & $\begin{array}{c}\text { Flow rate } 5 \% \\
\mathrm{SiH}_{4} \text { in } \mathrm{H}_{2} \\
(\mathrm{SCCM})\end{array}$ & $R$ & $\begin{array}{c}\text { Thickness } \\
(\mathrm{mm})\end{array}$ & $E_{\mathrm{G}}(\mathrm{eV})$ & $\begin{array}{c}\text { Deposition rate } \\
(\AA / \mathrm{s})\end{array}$ \\
\hline No. & 40 & 100 & 535 & 1.87 & 4.46 \\
1 & 20 & 200 & 494 & 1.90 & 4.12 \\
2 & 10 & 400 & 461 & 1.84 & 3.84 \\
3 & 6.4 & 630 & 160 & 2.16 & 1.32 \\
\hline
\end{tabular}

degradation after long term light soaking and the light-induced changes could be annealed at much lower temperature $\left(<150^{\circ} \mathrm{C}\right)$. The p-i-n solar cells with i-layer deposited using these conditions are likely to show better stability.

\section{Experimental}

Thin films of a-Si:H (about 160-535 nm) are deposited by RF PECVD technique on Corning 7059 glass substrates using the precursor gases hydrogen diluted silane (5\% silane in hydrogen) and argon in a load lock based chamber. The base pressure of the chamber has been better than $10^{-7}$ Torr. Prior to deposition, the argon flow rate is fixed so that a constant pressure of 0.355 Torr is achieved in the chamber without any silane flow. Silane flow rate is then varied in the range 6.4-40 SCCM to get different argon dilution ratios $(R)$. The substrate temperature and RF power for all the films are $300^{\circ} \mathrm{C}$ and $60 \mathrm{~W}$ respectively. While deposition, the pressure in the chamber is kept constant at 0.7 Torr without disturbing the argon and silane flow rate. The deposition conditions as well as the estimated thickness and band gap of the films are shown in table 1.

The films are structurally characterized by XRD, SEM and Raman spectroscopy respectively. The XRD studies are performed at grazing angle of incidence while for Raman studies excitation wavelength of $514.532 \mathrm{~nm}$ is used. Raman crystallinity fraction $\left(X_{\mathrm{c}}\right)$ is calculated by deconvoluting the spectrum into amorphous (480 $\left.\mathrm{cm}^{-1}\right)$, nanocrystalline $\left(\sim 520 \mathrm{~cm}^{-1}\right)$ and intermediate $\left(\sim 500 \mathrm{~cm}^{-1}\right)$ components and then taking the fractional area under the nanocrystalline peak. The thickness and optical constants of these films are estimated with the help of UV-Vis transmission measurements in the range of 400-1100 nm by following Swanepoel [9], while the band gap $\left(E_{\mathrm{G}}\right)$ of the films is estimated by plotting $\sqrt{\alpha h \nu}$ vs. $h \nu$ in the high absorption region. Photoluminescence (PL) studies are performed using excitation wavelength 300,350 and $375 \mathrm{~nm}$ respectively.

The transport and stability of the films are studied by conductivity measurements $\left(\sigma(T)\right.$, in the temperature range $\left.35-200^{\circ} \mathrm{C}\right)$ and light soaking. For $\sigma(T)$ measurements, silver paint electrodes (length $1 \mathrm{~cm}$, separation $\sim 1 \mathrm{~mm}$ ) are used 


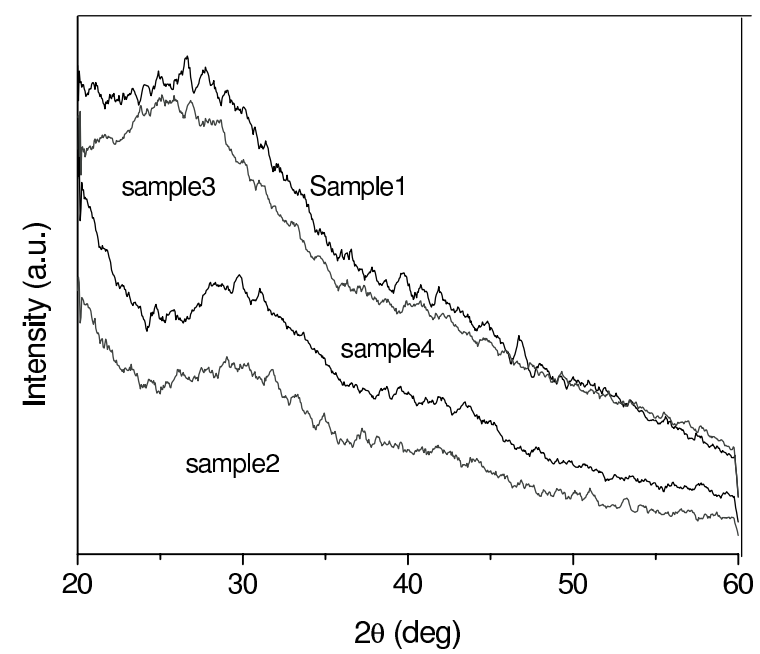

Figure 1. XRD pattern of the films prepared using argon dilution. All the films show amorphous nature.

in co-planar geometry. All the measurements including annealing (at $200^{\circ} \mathrm{C}$ ) and light soaking are done in vacuum better than $10^{-5}$ Torr. Prior to the $\sigma(T)$ measurements, the films are annealed at $200^{\circ} \mathrm{C}$ for $2 \mathrm{~h}$ and then slowly cooled in vacuum (here onwards this state will be referred to as SC state). A $100 \mathrm{~W}$ halogen lamp is used for photoconductivity measurements and also for light soaking in vacuum through an optical window. The intensity of light falling on the sample is $\sim 100 \mathrm{~mW} \mathrm{~cm}^{-2}$. Light soaking is done in vacuum in SC state of the films at room temperature using water filter to avoid heating of the films. A decrease in $\sigma_{\mathrm{d}}$ (dark conductivity) and $\sigma_{\mathrm{ph}}$ (photoconductivity) is seen for the first $2 \mathrm{~h}$ of light soaking after which it is saturated. The films are light soaked for $12 \mathrm{~h}$ (LS state). The $\sigma(T)$ measurements are done while heating and cooling in the SC as well as LS states.

\section{Results}

Figure 1 shows the XRD pattern of the films. The XRD data reveal the amorphous nature for all the samples. SEM studies also show smooth conchoidal surface morphology. From the cross-sectional SEM on the fractured surfaces of the films also no columnar structure is observed.

For argon dilution ratio $R \leq 400$, the deposition rate is about $4-5 \AA /$ s giving $460-540 \mathrm{~nm}$ thickness for the films for a deposition time of $20 \mathrm{~min}$. As $R$ is increased ( $\mathrm{SiH}_{4}$ flow rate is decreased) further, the deposition rate decreases drastically (1.3 $\AA / \mathrm{s}$, for $R=630$ ), resulting in a very low thickness of the film. Even for this argon dilution, the deposition rate is found to be much higher compared to that reported in the literature $(0.5-0.83 \AA / \mathrm{s})[5]$. For $R \leq 400$ the band gap is estimated to be $\sim 1.85-1.90 \mathrm{eV}$, which increases to $2.16 \mathrm{eV}$ for $R=630$. 
Purabi Gogoi, P N Dixit and Pratima Agarwal

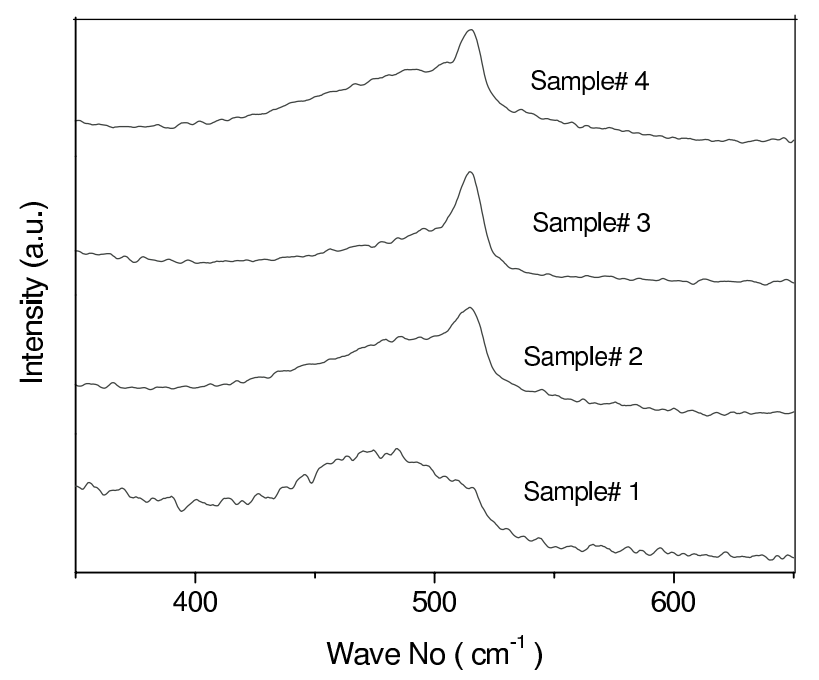

Figure 2. Raman scattering spectrum for the films. All the samples show a broad peak at $480 \mathrm{~cm}^{-1}$ corresponding to amorphous silicon and a sharp peak near $515 \mathrm{~cm}^{-1}$ corresponding to formation of nanocrystallites.

Raman studies show that the films are amorphous in nature with some nanocrystallites embedded in the network. Figure 2 shows the Raman data for the films. From the figure it is clear that the crystallinity increases with the dilution ratio. However, for the film with $R=630$, the crystalline fraction is lower than the previous film in the series which could be because of the low thickness of the film, as the first few layers of the films are amorphous in nature with isolated nanocrystalline nuclides. The detailed results of Raman studies are presented elsewhere [10]. The PL studies give broad weak intensity peaks in the range of 500-700 nm (figure 3), which could be due to the presence of nanocrystallites in the samples.

Room temperature dark conductivity of the SC films ranges from $\sim 10^{-10}$ to $10^{-6} \Omega^{-1} \mathrm{~cm}^{-1}$, while photoconductivity varies from $\sim 10^{-7}$ to $10^{-5} \Omega^{-1} \mathrm{~cm}^{-1}$. Figure 4 shows the variation of $\sigma_{\mathrm{d}}\left(35^{\circ} \mathrm{C}\right)$ and $\sigma_{\mathrm{ph}}\left(35^{\circ} \mathrm{C}\right)$ as a function of argon dilution ratio, in the SC and LS states. $\log \sigma_{\mathrm{d}}$ vs. $10^{3} / T$ curve for these films could be best fitted with two activation energies. Films prepared with the least argon dilution $(R=100)$ show a small value of $\sigma_{\mathrm{d}}$ with $\sigma_{\mathrm{ph}} / \sigma_{\mathrm{d}}$ ratio of more than four orders of magnitude, characteristic of a-Si:H films prepared with no dilution or small hydrogen dilution. When $R$ is increased to 200 , the formation of nanocrystallites begins and the films exhibit higher $\sigma_{\mathrm{d}}$ and significantly lower $\sigma_{\mathrm{ph}}$ (with $\sigma_{\mathrm{ph}} / \sigma_{\mathrm{d}} \sim 10$ only). For the films with $R=400$, which shows the maximum crystallinity fraction, $\sigma_{\mathrm{d}}$ becomes very low and $\sigma_{\mathrm{ph}} / \sigma_{\mathrm{d}} \sim 2 \times 10^{5}$ (figure 5 ). Though the film corresponding to $R=200$ exhibits less light-induced changes, the $\sigma_{\mathrm{ph}} / \sigma_{\mathrm{d}}$ ratio is small for these films. On the other hand, for the film with $R=400$, the light-induced changes are less and also the $\sigma_{\mathrm{ph}} / \sigma_{\mathrm{d}}$ value is quite high. The $\sigma_{\mathrm{d}}$ curve in this case $(R=400)$ has a nearly single activation energy $E_{\mathrm{d}} \sim 0.88 \mathrm{eV}$. These values correspond to very good quality a-Si:H films suitable for device applications. Further increase in argon dilution increases $\sigma_{\mathrm{d}}$ considerably with smaller activation energy. In this case, $\sigma_{\mathrm{d}}$ 


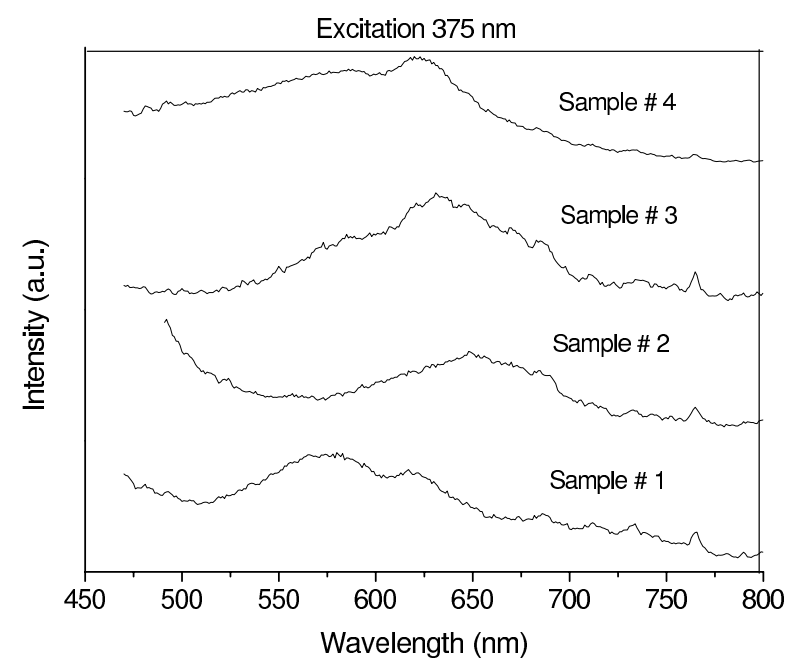

Figure 3. Photoluminescence emission spectrum of the samples under the present study. Weak and broad peaks are observed in the range 500-700 $\mathrm{nm}$. The figure shows the data for excitation wavelength $375 \mathrm{~nm}$.

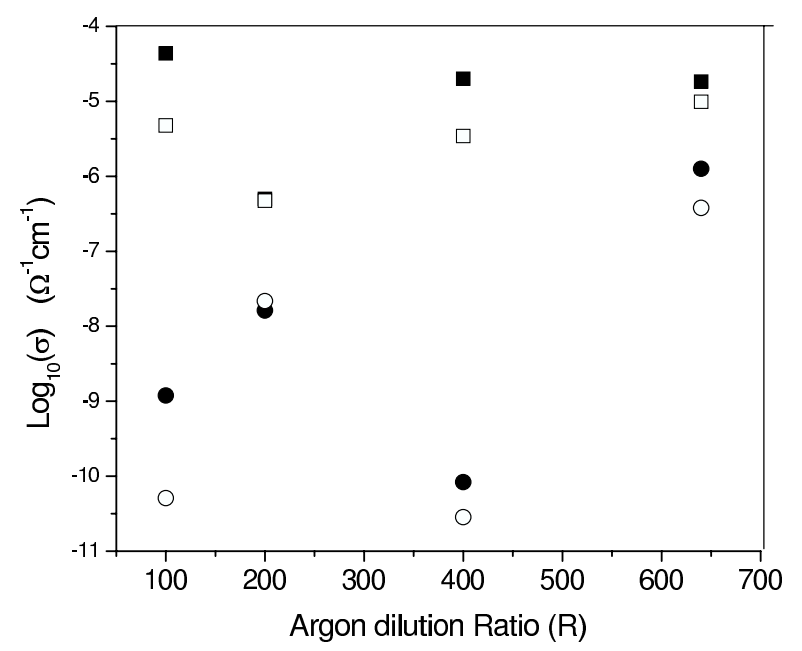

Figure 4. Variation of dark conductivity $\left(\sigma_{\mathrm{d}}\right)$ and photoconductivity $\left(\sigma_{\mathrm{ph}}\right)$ as a function of argon dilution ratio, $R$ in both SC state as well as LS state. Circles are for dark conductivity while squares are for photoconductivity. Filled symbols are for SC state and open ones for LS state. Errors are included in the size of the symbol.

is close to the microcrystalline films, which is also supported by drastic decrease in the deposition rate.

After light soaking for $12 \mathrm{~h}(\mathrm{LS}), \sigma_{\mathrm{d}}\left(35^{\circ} \mathrm{C}\right)$ and $\sigma_{\mathrm{ph}}\left(35^{\circ} \mathrm{C}\right)$ for these films are not affected much; the maximum change is one order of magnitude for the films 
Purabi Gogoi, P N Dixit and Pratima Agarwal

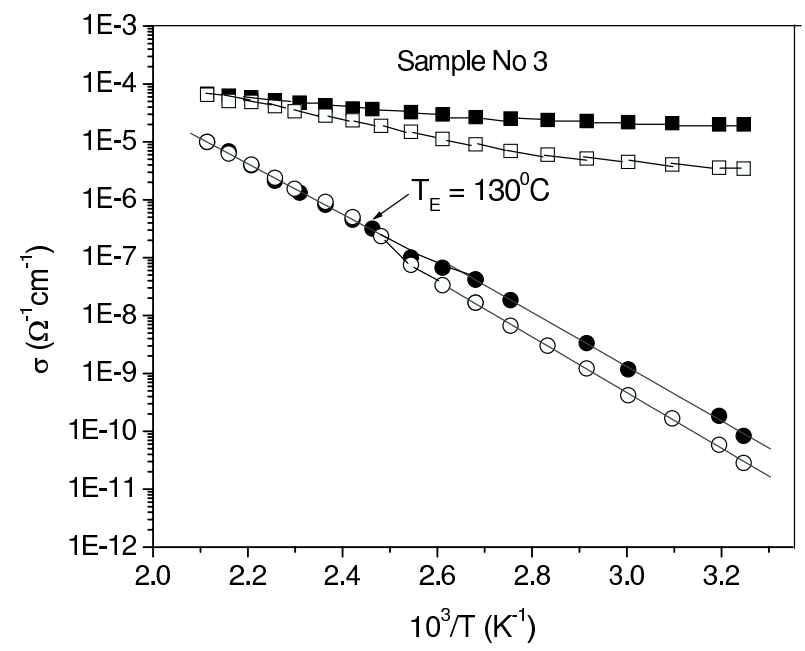

Figure 5. $\log _{10} \sigma$ vs. $10^{3} / T$ curve for sample no. 3 corresponding to argon dilution $R=400$ (silane flow rate $=10 \mathrm{SCCM}$ ). Circles are for dark conductivity while squares are for photoconductivity. The filled and open circles correspond to SC and LS states respectively. Dark conductivity curve for the two states meet at $T_{\mathrm{E}}$ and for $T>T_{\mathrm{E}}, \sigma(T)$ is the same for both the states.

corresponding to the lowest argon dilution. The $\sigma(T)$ curve in the LS state meets that in SC states at some equilibrium temperature $\left(T_{\mathrm{E}}\right)$ and for $T>T_{\mathrm{E}}, \sigma(T)$ is same for both the states. The changes in $\sigma_{\mathrm{d}}$ after light soaking and $T_{\mathrm{E}}$, for these films are much lower compared to that reported in literature (more than three orders of magnitude decrease and $\sim 200^{\circ} \mathrm{C}$ respectively) for undoped a-Si:H films. It is also observed that as the argon dilution increases, $T_{\mathrm{E}}$ decreases systematically. This lowering of $T_{\mathrm{E}}$ for these films may again be due to the improvement in MRO. Figure 6 shows $T_{\mathrm{E}}$ and $E_{\mathrm{d}}$ for $T>T_{\mathrm{E}}$ as a function of argon dilution. Conductivity measurements and stability studies suggest that the best quality a-Si:H films are produced when $R$ is close to 400 . The deposition rate for these samples is $3.84 \AA / \mathrm{s}$ and hence, these films are suitable for making cost-effective stable solar cells.

\section{Discussion}

In PECVD method, dilution by hydrogen or argon results in efficient dissociation of $\mathrm{SiH}_{4}$ into several species like $\mathrm{SiH}_{3}, \mathrm{SiH}_{2}$ with different lifetimes [8]. However, when only hydrogen is used as diluent gas, the main film forming precursor is $\mathrm{SiH}_{3}$ which has a less sticking coefficient and traverses long distance on the surface before finding a silicon dangling bond; also in this case the atomic hydrogen etches the growing surface, thus the deposition rate is lower. In the case of argon dilution other radicals like $\mathrm{Si}, \mathrm{SiH}, \mathrm{SiH}_{2}$ are present in the plasma. Due to their low mobility and high reactivity these species react with $\mathrm{Si}-\mathrm{H}$ bond on the surface resulting in columnar growth with high deposition rate $[4,5,11]$. 


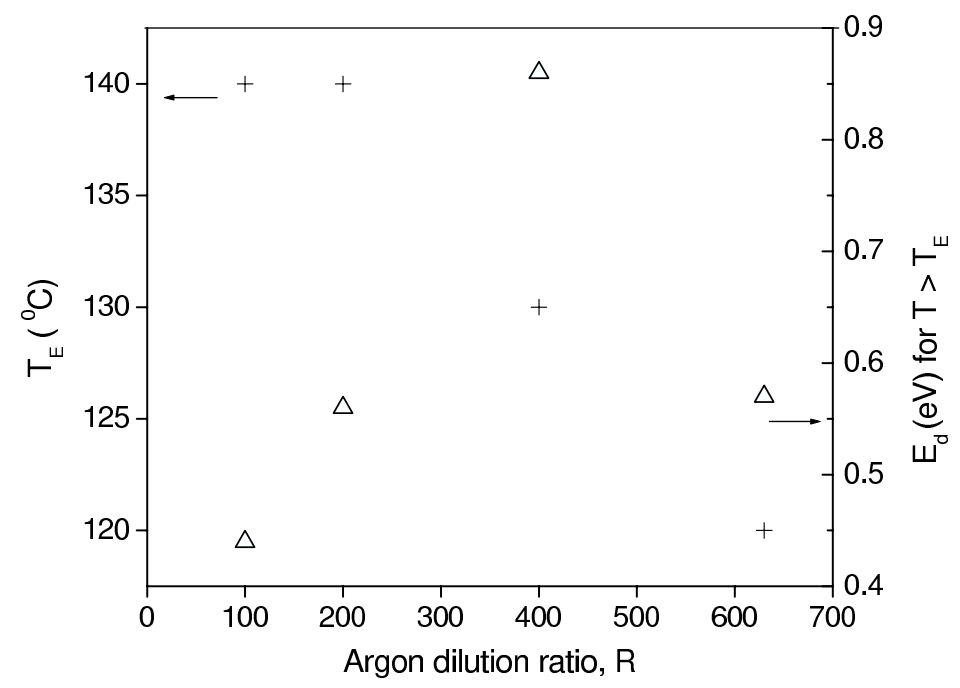

Figure 6. Variation of $T_{\mathrm{E}}$ and $E_{\mathrm{d}}$ as a function of argon dilution ratio. The plus are for $T_{\mathrm{E}}$ while triangles are for $E_{\mathrm{d}}$. Errors are included in the size of the symbol.

In our case, when both hydrogen and argon are used as diluents, the presence of atomic hydrogen and $\mathrm{Ar}^{*}$ causes effective dissociation of $\mathrm{SiH}_{4}$. The mobility of the $\mathrm{SiH}_{n}$ radicals increases considerably due to coverage of surface by hydrogen. The species therefore insert themselves at energetically favorable sites preventing columnar growth, thus improving MRO. Ar* also releases energy that is used in breaking weak $\mathrm{Si}-\mathrm{Si}$ bonds [12] and reconstructing strong $\mathrm{Si}-\mathrm{Si}$ bonds thus further improving the MRO and stability.

We associate the improved opto-electronic properties, better stability against light soaking and lower $T_{\mathrm{E}}$ for these films with the improved MRO. $T_{\mathrm{E}}$ decreases systematically with the increase in argon dilution, which indicates the lowering of defect annealing energy. The metastable changes after light soaking are believed to be due to the presence of potential fluctuations caused by inhomogeneous growth [13]. A mixture of hydrogen and argon dilution of silane possibly causes the homogeneous growth and improved order and thus less potential fluctuations in these films, which results in the reduced degradation of these films after exposure to light.

\section{Conclusion}

In this paper, we have reported the high deposition rate $(\sim 4-5 \AA / \mathrm{s})$, good transport properties and better stability against exposure to light of a-Si:H films prepared by argon and hydrogen dilution of $\mathrm{SiH}_{4}$. Presence of both $\mathrm{Ar}^{*}$ and atomic hydrogen in the plasma helps in the efficient dissociation of $\mathrm{SiH}_{4}$ and in the reconstruction of strong $\mathrm{Si}-\mathrm{Si}$ bonds thus improving the $\mathrm{MRO}$ and stability against light soaking. The best-quality samples are obtained with argon dilution ratio around 400 for 


\section{Purabi Gogoi, P N Dixit and Pratima Agarwal}

$\mathrm{SiH}_{4}$ diluted with hydrogen. The films prepared under these conditions are best suited for cost-effective and stable device applications.

\section{Acknowledgement}

The work reported in this paper is supported by a grant from CSIR, New Delhi, India.

\section{References}

[1] Technology and application of amorphous silicon edited by R A Street (Springer, Berlin, Germany, 1999)

[2] D L Staebler and C R Wronski, Appl. Phys. Lett. 31, 292 (1977)

[3] P Alpuim, V Chu and J P Conde, J. Appl. Phys. 86, 3812 (1999)

[4] J C Knights and R A Lujan, Appl. Phys. Lett. 35, 244 (1979)

[5] D Das, M Jana and A K Barua, J. Appl. Phys. 89, 3041 (2001)

[6] P P Ray, N D Gupta, P Choudhuri, D L Williamson, S Vignoli and C Longeaud, J. Non-Cryst. Solids 299-302, 123 (2002)

[7] P Choudhuri, R Meaudre and C Longeaud, J. Non-Cryst. Solids 338-340, 690 (2004)

[8] A Matsuda, J Non-Cryst. Solids 338-340, 1 (2004) and references therein

[9] R Swanepoel, J. Phys. E16, 1214 (1983)

[10] P Gogoi, P N Dixit and P Agarwal, Solar Energy Materials and Solar Cells 91, 1253 (2007)

[11] R A Street, Hydrogenated amorphous silicon (Cambridge University Press, Cambridge, UK, 1992) ch. 2

[12] M Jana, D Das and A K Barua, J. Appl. Phys. 91, 5442 (2002)

[13] P Agarwal and S C Agarwal, Phil. Mag. B80, 1327 (2000) 